\title{
Pain and Pharmacologic Pain Management in Long-Stay Nursing Home Residents
}

Jacob N Hunnicutt MPH, ${ }^{a}$ Christine M Ulbricht PhD, ${ }^{a}$ Jennifer Tjia MD MS, ${ }^{a}$ Kate L Lapane PhD $\mathrm{MS}^{\mathrm{a}}$

${ }^{a}$ Department of Quantitative Health Sciences, University of Massachusetts Medical School, Worcester, MA 01655

\section{Corresponding Author:}

Jacob N. Hunnicutt, MPH

Address: Department of Quantitative Health Sciences, University of Massachusetts Medical

School, 55 Lake Road North, Worcester MA 01655, USA

Email: Jacob.Hunnicutt@umassmed.edu

Phone: $508-856-8798$

Fax: 508-856-8993

Funding Sources: This work was funded by the following NIH grants: 1TL1TR001454, 1R56NR015498-01, and R21CA198172-02.

Running head: Pain in the nursing home

Number of pages: 29

Number of tables: 2

\section{Number of figures: 2}

Word count: 4,298 


\section{ABSTRACT}

Prior studies estimate that $>40 \%$ of long-stay nursing home $(\mathrm{NH})$ residents experience persistent pain, with $20 \%$ of residents in pain receiving no analgesics. Strengthened $\mathrm{NH}$ surveyor guidance and improved pain measures on the Minimum Data Set (MDS) 3.0 were introduced in March 2009 and October 2010, respectively. This study aimed to provide estimates after these important initiatives of: 1) prevalence and correlates of persistent pain; and 2) prevalence and correlates of untreated or undertreated persistent pain.

We identified 1,387,405 long-stay residents in United States NHs between 2011-2012 with 2 MDS assessments 90 days apart. Pain was categorized as persistent (pain on both assessments), intermittent (pain on either assessment), or none. Pharmacologic pain management was classified as untreated pain (no scheduled or as needed medications received) or potentially undertreated (no scheduled received). Modified Poisson models adjusting for resident clustering within $\mathrm{NHs}$ provided adjusted prevalence ratios estimates (APR) and $95 \%$ confidence intervals $(\mathrm{Cl})$.

The prevalence of persistent and intermittent pain was $19.5 \%$ and $19.2 \%$ respectively but varied substantially by age, gender, race/ethnicity, cognitive impairment, and cancer. Of residents in persistent pain, $6.4 \%$ and $32.0 \%$ were untreated or undertreated. Racial/ethnic minorities (nonHispanic blacks vs. whites, APR $=1.19,95 \% \mathrm{Cl}$ : 1.13-1.25) and severely cognitively impaired residents (severe vs. no/mild APR $=1.51,95 \% \mathrm{Cl}: 1.44-1.57$ ) had an increased prevalence of untreated and undertreated pain.

One in five $\mathrm{NH}$ residents has persistent pain. Although this estimate is greatly improved, many residents may be undertreated. The disturbing disparities in untreated and undertreated pain need to be addressed.

Keywords: Nursing home, Epidemiology, Persistent Pain, Pharmacologic pain managementPersistent Pain, Pharmacologic pain management 


\section{INTRODUCTION}

More than 1.4 million adults in the United States (US) live in nursing homes $(<0.001 \%$ of the US population $<65$ years old; $2.8 \%$ of the US population $>65$ years old; and $10.4 \%$ of the US population $>85$ years old). [3] This care setting is particularly important to the most vulnerable among us including the oldest old ( $\geq 85$ years), those with moderate to severe cognitive and/or functional impairment, and the dying.[5,44] As the population continues to age, this setting will become increasingly important for the care of these vulnerable populations.

Essential to residents' quality of life is the appropriate management of pain, which affects $35 \%$ of all nursing home residents.[3] Historically, pain has been untreated or undertreated in US nursing homes, with approximately $20 \%$ of all residents experiencing malignant or nonmalignant pain receiving no pharmacologic pain management.[2,51,52] However, previous studies of pain and pain management may not be applicable to current practice in US nursing homes because they preceded Centers for Medicare and Medicaid Services (CMS) policy changes including strengthened nursing home surveyor guidance in 2009 (F-tag 309), and preceded the 2010 introduction of Minimum Data Set (MDS) 3.0 with enhanced pain measures. [2,8,18,20,23,31,36,38,40,43,45,51-53] Declining trends in the prevalence of moderate to severe pain between 2006 and 2009 are encouraging.[8,40] Yet, questions remain on the overall burden of pain and the degree to which it may be currently untreated or potentially undertreated in US nursing homes.[40]

The Institute of Medicine called for "better data" to enhance the understanding of pain and pain management strategies in vulnerable populations, such as nursing home residents.[16] Therefore, using comprehensive, national data from 2011-2012 of all long-stay US nursing home residents, we conducted this study to estimate: 1) the prevalence and correlates of intermittent and persistent pain; and 2) the prevalence and correlates of untreated or potentially 
undertreated persistent pain in US nursing home residents. Although we had no prior hypotheses on the overall burden of pain, we did hypothesize that older residents, men, racial/ethnic minorities, and cognitively impaired residents would have a higher prevalence of untreated and potentially undertreated pain.

\section{METHODS}

\section{Study Design and Data Source}

We used routinely-collected administrative data from 2011-2012 to conduct a crosssectional study. We created our cohort using the Minimum Data Set 3.0, a federally required, standardized assessment of all nursing home residents living in Medicare- or Medicaid-certified homes (approximately $96 \%$ of all nursing homes). The MDS 3.0 includes more than 450 items to assess residents' overall health including pain, cognition, mood, functional status, medical conditions, and other measures.[35,36,38] Comprehensive MDS 3.0 assessments are completed by registered nurses at admission, annually, and whenever there is a significant change in status. Condensed quarterly assessments are conducted at 90 day intervals between comprehensive assessments.

The University of Massachusetts Medical School Institutional Review Board approved this study.

\section{Study Sample}

We identified a sample of long-stay nursing home residents during 2011-2012, defined as residents with: 1) >100 cumulative days in the same nursing home with no gaps in residency $>30$ days; and 2) a comprehensive or quarterly MDS assessment in the study period $(n=1,697,384)$. We focused on long-stay residents because they require long-term assistance to manage their chronic disabilities (average length of stay of $\sim 2$ years). [49] In contrast, short-stay 
residents or those receiving skilled nursing facility (SNF) care are predominately recovering from acute illnesses and are normally in the nursing home for a limited period of time before being discharged to the community (average length of stay of $\sim 40$ days). [49]

Eligible long-stay residents had to have a comprehensive or quarterly MDS assessment (the index assessment) that was: 1) preceded by a prior (comprehensive or quarterly) MDS assessment $90+/-20$ days before the index assessment; and 2) had a comprehensive assessment within 180 days of the index assessment $(98,311$ with no prior assessment, 85,336 with no comprehensive MDS assessment). These study design criteria were necessary because residents without a comprehensive or quarterly assessment had limited information on pain and other important study variables, residents with no prior MDS assessment $90+/-20$ days before the index assessment could not be assessed for persistent pain (pain present on two MDS assessments approximately three months apart, described in detail below), and residents without a comprehensive assessment were missing data on important potential causes of pain (e.g., cancer, arthritis, and osteoporosis) that were not available on the condensed, quarterly MDS assessments. We additionally excluded long-stay residents who received SNF care at or within 30 days of the index date because of differences between those needing SNF care and other long-stay residents highlighted above $(n=24,947)$.[49] For the remaining eligible residents, we randomly chose an index assessment for those with multiple eligible assessments $(1,488,790$ unique residents). Of these residents, we further excluded those who were comatose $(n=5,194),<18$ years old $(n=1,436)$, or missing data on important covariates $(n=94,755)$. The final analytic sample included 1,387,405 residents. See eFigure 1 in supplemental appendix for more detail. 


\section{Pain and Pain Management}

On the MDS 3.0, all measures of pain are included on quarterly or comprehensive assessments. Prior studies that compared the inter-rater agreement between facility nurses and gold-standard research nurses found that these items have excellent reliability. $(K>0.96)$.[35] We first categorized the presence of pain at the index and preceding MDS assessment. For each assessment, the registered nurse administering the MDS asked residents who were cognitively and physically able to self-report ( $\geq 90 \%$ of all long-stay residents)[46] on the presence of pain in the 5 days before each assessment. For residents who self-reported pain, information on frequency (rarely, occasionally, frequently, or constantly) and severity of the worst pain in the lookback period was collected. Residents rated pain severity using either the Verbal Descriptor Scale (categorizing pain as mild, moderate, severe, or very severe/horrible) or the Numeric Pain Scale (categorizing pain from 0 [no pain] to 10 [very/severe horrible pain]). A validated crosswalk was used to compare the two scales.[7] We used the Centers for Medicare and Medicaid Services (CMS) quality indicator definition of pain to categorize self-reporting residents as having no pain, mild/infrequent pain (mild to severe pain occurring rarely or occasionally), or moderate/severe pain (moderate/severe pain occurring frequently or almost constantly or very severe/horrible occurring at any frequency).[3] For residents who could not self-report their pain, staff review the medical record, consult other staff, and directly observe the resident to ascertain indicators of pain (e.g., crying, moaning, grimacing) and frequency of pain in the previous five days. We categorized staff-assessed pain as no pain, infrequent pain (1-2 days), or frequent pain occurring $\geq 3$ days.[15] For our analyses, we combined self-reported moderate/severe pain and staff-assessed frequent pain, self-reported mild/infrequent pain and staff-assessed infrequent pain, and no self-reported pain and no staff-assessed pain. 
We were conceptually interested in the prevalence of persistent pain, defined according to prior guidelines as pain with a duration $\geq 3$ months.[1] To operationally define persistent pain, we evaluated the index and preceding assessments. Residents were considered to have persistent pain if pain was documented at both assessments. We categorized residents as having intermittent pain if they had pain at either (but not both) assessments. Residents with no pain documented on both assessments were categorized as having no pain.

We assessed whether residents received pharmacologic pain management at the index assessment using MDS 3.0 J0100. These items have excellent reliability in a study of inter-rater agreement between gold-standard research nurses and facility nurses ( $K>0.92)$. [35] Nurses record whether the resident received a scheduled pain regimen or as needed (pro re nata, PRN) pain medication in the five days prior to the assessment based on medical record review. We defined residents as having untreated pain if they experienced any pain at the index assessment or had persistent pain and received no pharmacologic pain management. For those in persistent pain, we considered residents as having potentially undertreated pain if they did not receive scheduled analgesics or PRN medications as recommended by treatment guidelines for malignant and nonmalignant pain. $[1,11]$

\section{Resident Characteristics}

We evaluated resident characteristics that may influence pain ascertainment or receipt of analgesics based on prior literature.[2,8,23,31,51] This included age ( $<65$ years, $65-74$ years, 75-84 years, >85 years), gender, race/ethnicity (non-Hispanic White, non-Hispanic Black, Hispanic, other), physical functioning, cognitive functioning, whether the resident rejected care in the previous 7 days (e.g., taking medications, activities of daily living [ADL] assistance), hospice use in the previous 14 days, and active comorbidities associated with pain (e.g., fractures, arthritis, osteoporosis). We categorized residents' cognitive impairment using CMS 
definitions based on either the Brief Interview of Mental Status (BIMS; range 0-15) for residents who could self-report or the Cognitive Performance Scale (CPS; range 0-6) for those unable to self-report.[3,25,37] Residents were categorized as having no or mild impairment (BIMS 13-15 or CPS 0-2), moderate impairment (BIMS 8-12 or CPS 3-4), or severe impairment (BIMS 0-7 or CPS 5-6). We categorized physical functioning using the MDS-ADL Self-Performance Hierarchy (range 0-7) to categorize residents as totally dependent (5-6), extensively dependent (3-4) or no to limited impairment (0-2).[26]

\section{Analysis}

We conducted descriptive analyses to describe the overall prevalence of persistent and intermittent pain by severity/frequency of pain reported at both the preceding and index MDS assessments. We conducted stratified analyses to examine variation in persistent and intermittent pain by demographics, cognitive impairment, and cancer. We evaluated correlates of intermittent and persistent pain (while adjusting for potential confounders) using multinomial logistic models with robust variance estimation to calculate adjusted odds ratios (AOR) and 95\% confidence intervals $(\mathrm{Cl})$ that accounted for the clustering of $\mathrm{NH}$ residents within facilities.[50]

We described pharmacologic pain management strategies by the level of pain reported on the index assessment and persistent pain to quantify the prevalence of untreated pain. For the subset of residents in persistent pain, we estimated the association between resident characteristics and being pharmacologically untreated or potentially undertreated using modified Poisson models with robust variance estimation to calculate adjusted prevalence ratios (APR) with $95 \% \mathrm{Cl}$ while accounting for the clustering of $\mathrm{NH}$ residents within facilities. $[55,56]$ 
Because of our large sample size, we did not use statistical significance as a threshold to evaluate whether our results were clinically significant.[13] Rather, we considered effect estimates $\geq 10 \%$ (AORs or APRs $\geq 1.10$ or $\leq 0.90$ ) as being potentially relevant to clinicians and policymakers.[43]

We conducted several additional analyses to examine potentially meaningful differences in pain reporting and pharmacologic pain management in our study population. First, we stratified by cancer status to investigate whether there were differences in pain prevalence, general pharmacologic management, and correlates of untreated or undertreated pain due to cancer status and source of pain. Although general pain management strategies should not differ by cancer status (e.g., residents should receive medication - with preference for scheduled analgesics - to manage persistent pain regardless of whether pain is due to cancer or not), we conducted these analyses to compare our results to prior studies that restricted sample to residents with malignant or nonmalignant pain.[2,8,31,51,52] Second, we conducted sensitivity analyses examining whether correlates of untreated and potentially undertreated pain remained after restricting our sample to those reporting moderate-to-severe/frequent pain only. We wanted to know if the potential burden of untreated and undertreated pain would be the same in this restricted population in the most pain and compare these results to a prior study that used a similar operational definition.[8] Finally, we conducted sensitivity analyses to examine pain prevalence, pharmacologic management, and correlates of untreated or potentially undertreated pain after restricting our sample to those who were able to self-report their own pain and were not severely cognitively impaired. These analyses address concerns of potential differences in pain ascertainment by cognitive status and aid in comparing our results to previous studies that used similar restrictions. $[8,51,52]$ 


\section{RESULTS}

More than one-third of long-stay nursing home residents experienced intermittent (19.1\%) or persistent pain (19.5\%) over the 3-month study period. A small proportion of residents (4.2\%) had documented moderate-to-severe/frequent pain at both assessments. Of those with initial reports of moderate-to-severe/frequent pain $(n=155,174), 27.4 \%$ had mild and $35.1 \%$ had no pain documented at the next assessment. Conversely, $5.1 \%$ and $16.7 \%$ of those experiencing no or mild/infrequent pain at their first assessment reported moderate/severe pain at the second assessment, respectively.

Figure 1 shows there were differences in the unadjusted prevalence of overall pain and persistent pain by resident age, gender, race/ethnicity, cognitive impairment, and cancer status. Older residents had less documented pain than younger residents, with the oldest old reporting substantially less overall (35.5\% vs. $44.3 \%)$ and persistent pain $(15.9 \%$ vs. $27.1 \%)$ than residents 18-64 years old. Men reported less overall pain than women (35.3\% vs. $40.2 \%)$. Racial/ethnic minorities reported less overall ( $31.3 \%$ vs. $40.6 \%)$ and persistent pain (14.9\% vs. $20.7 \%$ in comparison to non-Hispanic white residents. Moderately and severely cognitively impaired residents reporting lower levels of overall pain (moderate: $38.2 \%$; severe: $27.4 \%$ ) and persistent pain (moderate: 18.4\%; severe: 10.5\%) than residents with no/mild cognitive impairment (overall pain: 56.1\%; persistent pain: 34.1\%). Residents with cancer reported more overall pain than residents without cancer (44.5\% vs $38.2 \%)$, though differences in persistent pain $(22.9 \%$ vs. $19.2 \%)$ were less pronounced.

Table 1 shows that the differences noted in Figure 1 continued to be largely associated with documented persistent and intermittent pain after adjusting for other resident characteristics. In particular, racial/ethnic minorities reported less persistent and intermittent pain (relative to no pain), with non-Hispanic blacks reporting less persistent ( $A O R=0.66,95 \%$ 
$\mathrm{Cl}$ : 0.63-0.69) and intermittent pain (AOR=0.75, 95\% Cl: 0.73-0.76) than non-Hispanic whites. Additionally, cognitive impairment was strongly inversely associated with persistent (severely impaired vs. no impairment: $A O R=0.26,95 \% \mathrm{Cl}: 0.25-0.26)$ and intermittent pain (severely impaired vs. no impairment: $\mathrm{AOR}=0.48,95 \% \mathrm{Cl}: 0.47-0.49)$. Additional factors that increased the odds of being in intermittent or persistent pain included rejecting care (persistent pain only), hospice use, arthritis, fractures, pressure ulcers, and surgical wounds. Other markers of cognitive impairment including dementia and staff-assessed pain (persistent pain only) were inversely associated with documented pain.

Figure 2 shows that untreated pain was uncommon with $8.9 \%$ and $6.6 \%$ of residents in any pain at the index assessment and persistent pain receiving no pharmacologic pain management, respectively. Nearly one-third of those in persistent pain (32.0\%) received no scheduled analgesics. Scheduled analgesics were used by $34.4 \%$ of residents reporting no pain at index, $63.2 \%$ of those reporting any pain (mild/infrequent pain: $63.8 \%$; moderate-tosevere/frequent pain: $62.1 \%$ ) at index, and in $68.0 \%$ of those in persistent pain.

Table 2 shows the correlates of untreated and potentially undertreated persistent pain in our adjusted statistical models. Being older (e.g., $\geq 85$ years vs $65-74$ years: APR $=1.42,95 \% \mathrm{Cl}$ : 1.35-1.49), non-Hispanic black or other race/ethnicity vs. non-Hispanic white (non-Hispanic black: APR=1.19, 95\% Cl: 1.13-1.25; other: APR=1.15, 95\% Cl: 1.03-1.29), and cognitively impaired vs. no/mild impairment (moderate impairment: APR=1.24, 95\% CI: 1.19-1.29; severe impairment: APR=1.51, 95\% Cl: 1.44-1.57) were strongly associated with untreated pain. Alternatively, women were less likely than men to receive no pharmacologic pain management (APR=0.76, 95\% Cl: $0.74-0.79)$. Other factors associated with a reduced prevalence of untreated pain included receipt of hospice care, having cancer, arthritis, fractures, pressure ulcers, or surgical wounds. Other markers of cognitive impairment such as dementia were also associated with a higher prevalence of untreated pain. When examining correlates of potentially 
undertreated pain (not receiving scheduled analgesics), residents who were a racial/ethnic minority vs. non-Hispanic white (non-Hispanic black: APR=1.23, 95\% Cl: 1.20-1.25; Hispanic: $\mathrm{APR}=1.18,95 \% \mathrm{Cl}=1.14-1.22$; other: $\mathrm{APR}=1.11,95 \% \mathrm{Cl}: 1.05-1.17)$, those with moderate to severe cognitive impairment vs. those with no/mild cognitive impairment (moderate: APR=1.11, 95\% Cl: 1.09-1.12; severe: APR=1.14, 95\% Cl: 1.11-1.16), and those with fractures (vs. no fractures) or surgical wounds (vs. no surgical wounds) had a higher prevalence of potentially undertreated pain. Alternatively, residents receiving hospice care (vs. no hospice care), and those with arthritis (vs. no arthritis), osteoporosis (vs. no osteoporosis), or pressure ulcers (vs. no pressure ulcers) had a lower prevalence of potentially undertreated persistent pain.

In analyses stratified by cancer status and demographics/cognitive impairment, residents with cancer had more overall persistent $(+3.7 \%$ on average) and intermittent $(+2.6 \%$ on average) pain than residents with nonmalignant pain (eTable 1 in Supplementary Appendix). Younger persons with cancer reported far greater levels of persistent pain than those with nonmalignant pain (e.g., residents with cancer, 18-64 years old: 35.4\%; residents with nonmalignant pain, 18-64 years old: $25.8 \%$ ). Those with cancer were less likely to have untreated or potentially undertreated pain at the index assessment in comparison to those with nonmalignant pain (eTable 2-3). Risk factors for untreated and potentially undertreated pain were similar regardless of whether the resident experienced malignant or nonmalignant pain (eTable 4-5). Analyses restricted to those in the most severe pain did not substantially alter our finding on the prevalence and correlates of untreated/undertreated pain (eTable 6-7). When we restricted our sample to those who were able to self-report pain and were not severely cognitively impaired, the prevalence of persistent pain and intermittent pain increased to $28.1 \%$ and $20.5 \%$, respectively (eTable 8 ). However, the prevalence and correlates of untreated or potentially undertreated persistent pain did not substantially change (eTable 9-11). 


\section{DISCUSSION}

In 2011-2012, 38.6\% of long-stay NH residents experienced any pain, with nearly one in five residents having persistent pain. Among those in persistent pain, $6.4 \%$ received no pharmacologic pain management and over $30 \%$ received no scheduled analgesics. Our estimates of persistent pain prevalence in long-stay nursing home residents are substantially lower than historical estimates that range from to $41-49 \%$.[45,52,53] Given that many of the older studies use data $>15$ years old,[45,52,53] possible explanations for the lower pain prevalence in our study may include: 1) heightened attention to pain treatment through introduction of pain quality indicators (2002);2)strengthened surveyor guidance that holds nursing homes accountable for assessing and treating pain (F309; 2009) [18]; 3) improved measures in MDS 3.0 that emphasize "the resident voice" and highlight previously unmet needs in pain management $(2010)[36,46]$; 4) change in payment for analgesics with the introduction of Medicare Part D implementation that made medications more affordable for Medicare beneficiaries (2006); and 3) the growth of hospice and palliative careduring the last 15 years in this care setting.[24] It is unclear to what extent individual policy and culture changes may have potentially lowered pain prevalence, though studies and CMS reports have documented declines in reported pain between 2006 and 2013.[3,4,40] We believe that study design issues only partially explain differences in our estimate of persistent pain in comparison to prior national studies. Such differences include differing operational definitions of persistent pain, our inclusion of residents with severe cognitive impairment or those unable to self-report, and changes in pain ascertainment between MDS 2.0 and 3.0.[8,40,45,52,53] Sensitivity analyses intended to mimic restrictions used in prior research demonstrated that the substantial reductions in overall persistent pain were not due to our study design decision-making. 
Despite large reductions in the absolute prevalence of persistent pain, we found demographic and clinical trends in the prevalence of pain that are consistent with prior US research.[2,8,19,31,40,43,51,52]. In particular, younger residents, women, non-Hispanic whites, those with cancer, and those with no or mild cognitive impairment reporting higher levels of pain. Older adults, racial and ethnic minorities, and men may systematically underreport their pain, potentially due to cultural pressure to be stoic and intimidation to report their pain to health professionals.[12,14,17,27,28,39,54] Our estimates of pain may be conservative in these subgroups. Among residents with moderate or severe cognitive impairment, reports of pain were much lower than in those with no or mild impairment. It is unclear if pain was appropriately assessed in this group. Prior studies indicate that pain can be appropriately assessed in many residents with moderate to severe impairment, $[10,21,29,30]$ and the majority of long-stay residents do self-report.[46] However, excluding residents with severe cognitive impairment or those who were unable to self-report dramatically increased our estimates of persistent pain prevalence from $19.5 \%$ to $28.1 \%$. Continued efforts to improve pain ascertainment in such vulnerable $\mathrm{NH}$ residents is appropriate.

We found that $6.4 \%$ of long-stay nursing home residents in persistent pain received no pharmacologic pain management. This estimate is significantly lower than the $24.5 \%$ reported by Won et al., 2004 using data from 1998-2000 and 16.7\% reported by Fain et al., 2016 using 2008 data. [8,52] Potential decreases in untreated pain prevalence could be due to the policy changes highlighted above, though the relative influence of each policy change is unknown. Alternatively, prior studies using MDS 2.0 either measured analgesic use based on nursing staff reporting or Part D claims, which may results in some misclassification of pain medication use, especially for commonly prescribed over-the-counter medications (e.g., acetaminophen) not covered by Part D.[41] Although reductions in untreated pain appear to have occurred, current pain management practices may still be suboptimal. More than one-third of long-stay residents 
in persistent pain received no scheduled analgesics despite their recommended use in pain management guidelines.[1] It is unclear from our data what specific medications are being used to manage pain in long-stay residents.

Disparities in pharmacological management of persistent pain continue in the US. Older nursing home residents, non-Hispanic blacks, and those with cognitive impairment and/or dementia diagnosis had a higher prevalence of untreated pain. Prior studies conducted over the past 18 years have also documented these gaps in untreated pain prevalence.

$[2,8,20,23,31,51,52]$ When examining potentially undertreated pain, racial/ethnic disparities increased with non-white residents in persistent pain receiving less scheduled analgesics than non-Hispanic white residents. It is unclear why these disparities continue, though others have suggested that they may be due to potential implicit bias in healthcare providers. $[8,34]$ The time has come to conduct research on how best to promote equity in pain management in this vulnerable population. Some clinical characteristics were associated with a decreased prevalence of untreated or potentially undertreated pain. In general, residents in persistent pain with comorbidities known to cause pain (e.g., arthritis, fractures) had a lower prevalence of untreated or undertreated pain, though residents with fractures or surgical wounds were less likely to receive scheduled analgesics than other residents. This discrepancy may be due to the acuteness of these injuries. However, given concern that acute pain may be a significant cause of persistent pain if not properly treated,[1] further research is needed to understand how these types of injuries are treated over time in long-stay residents. In addition to painful comorbidities, receipt of hospice care was associated with a lower prevalence of untreated and potentially undertreated persistent pain. Hospice may give residents access to superior palliative care in comparison to traditional nursing home care, and prior studies document a strong association between hospice care and receipt of pharmacologic pain management.[15,23] Yet, these services are only available to residents with terminal diagnoses who elect hospice. Further 
interventions and policies may be needed to disseminate palliative care practices into a care setting that has traditionally prioritized improving and maintaining resident function over holistic resident-centered care.

Although our discussion has focused on the US context, pain and pain management in nursing home is an international issue.[47] A systematic review identifying studies from Australia, Canada, China, Taiwan, Singapore, Iran, Norway, the Netherlands, and the United Kingdom found pain prevalence to be highly prevalent, ranging from 27.8-79.5\%.[42] Further, in many cases pain management was not provided or inadequate, particularly for those with cognitive impairment. Similar trends in international pain prevalence and untreated/undertreated pain have also been reported.[22] This study has several strengths. First, we used comprehensive, national data from 2011-2012 that included more than 1.3 million long-stay nursing home residents. Our results are generalizable to nursing home residents in Medicare- or Medicaid certified homes. Second, through the MDS 3.0 we had access to enhanced pain assessments not available in previous national samples examining pain management. Third, we were able to measure analgesic use using the MDS 3.0. Because this is based on medical record review, it may more accurately capture medication use that may not be included in pharmaceutical claims such as over-the-counter medication.[41] However, this study also had limitations. We could only assess pain when the resident had an MDS assessment (required every 90 days). We lacked information on pain between assessments. Additionally, our study design excluded long-stay residents without prior assessments or comprehensive assessments within 180 days of the index assessment and those with missing data. Although our results may not be generalizable to these residents, further examination of their reported pain suggests that their exclusion did not substantially alter study findings. We also note large differences in pain reporting between those with no/mild impairment and more severe cognitive impairment, we were concerned that there may be under-ascertainment of pain. However, prior studies 
emphasize that even those with severe cognitive impairment can still accurately self-report pain.[10,21,29,30] We conducted sensitivity analyses restricting our sample to those without severe cognitive impairment or unable to self-report - overall prevalence of pain was affected but correlates of untreated and potentially undertreated pain were similar to primary analyses. We also lacked detailed information on specific medications used, so we could only comment on general pain management strategies recorded on the MDS. Finally, we specifically focused on pharmacologic interventions, but non-pharmacologic interventions (e.g., physical therapy, nerve blocks, electrical stimulation) are also important in the management of pain. $[33,48]$

In conclusion, we found that the prevalence of persistent pain and untreated persistent pain were dramatically lower than previously reported estimates from data sources predating major national initiatives that could potentially influence the ascertainment and treatment of pain. However, many residents in persistent pain may still be undertreated, as the use of scheduled analgesics was low despite guideline recommendations for their use. Previously documented disparities in treatment are still present and require attention to improve the quality of nursing home care for all residents. Further work is needed to characterize the appropriateness of current pain management practices, as nursing homes have a documented history of poor pain management practices beyond underuse,[6,9,32] and the overall prevalence of pain is still high despite improvements.

\section{ACKNOWLEDGMENTS}

Conflicts of Interest: JT is consultant with CVS Caremark for the National Pharmacy and Therapeutics Committee. All other authors: None.

JNH was supported by NIH grant 1TL1TR001454.

CMU was supported by NIH grants 1R56NR015498-01, and R21CA198172-02.

JT was supported by NIH grant R56NR015498-01, R21CA198172-02, and a 
Sojourns Scholar Leadership Award from the Cambia Health Foundation.

KLL was supported by NIH grants 1TL1TR001454, 1R56NR015498-01, and R21CA198172-02.

\section{REFERENCES}

[1] American Geriatrics Society Panel on Pharmacological Management of Persistent Pain in Older Persons. Pharmacological management of persistent pain in older persons. J Am Geriatr Soc 2009;57:1331-46.

[2] Bernabei R, Gambassi G, Lapane K, Landi F, Gatsonis, C, Dunlop R, Lipsitz L, Steel K, Mor V. Management of pain in elderly patients with cancer. SAGE Study Group.

Systematic Assessment of Geriatric Drug Use via Epidemiology. JAMA 1998;279:187782.

[3] Centers for Medicare and Medicaid Services. Nursing Home Data Compendium, 2015 Ed. [on-line]. Available at https://www.cms.gov/Medicare/Provider-Enrollment-andCertification/CertificationandComplianc/Downloads/nursinghomedatacompendium_5082015.pdf. Accessed December 5, 2016.

[4] Centers for Medicare and Medicaid Services. Nursing Home Data Compendium, 2013 Ed. [on-line]. Available at https://www.cms.gov/Medicare/Provider-Enrollment-andCertification/CertificationandComplianc/downloads/nursinghomedatacompendium_508.pd f. Accessed December 5, 2016.

[5] David S, Sheikh F, Mahajan D, Greenough W, Bellatoni M. Whom do we serve? Describing the target population for post-acute and long-term care, focusing on nursing facility settings, in the era of population health. J Am Med Dir Assoc 2016;17:574-80.

[6] Dosa DM, Dore DD, Mor V, Teno JM. Frequency of long-acting opioid analgesic initiation in opioid-naïve nursing home residents. J Pain Symptom Manage. 2009;38:515-21. 
[7] Edelen MO, Saliba D. Correspondence of verbal descriptor and numeric rating scales for pain intensity: an item response theory calibration. J Gerontol A Biol Sci Med Sci 2010;65:778-85.

[8] Fain KM, Alexander GC, Dore DD, Segal JB, Zullo AR, Castillo-Salgado C. Frequency and predictors of analgesic prescribing in US nursing home residents with persistent pain. J Am Geriatr Soc 2016. Published online ahead of print.

[9] Fain KM, Castillo-Salgado C, Dore DD, Segal JB, Zullo AR, Alexander GC. Inappropriate Fentanyl Prescribing Among Nursing Home Residents in the United States. J Am Med Dir Assoc 2016. Published online ahead of print.

[10] Fisher SE, Burgio LD, Thorn BE, Hardin JM. Obtaining self-report data from cognitively impaired elders: methodological issues and clinical implications for nursing home pain assessment. Gerontologist 2006;46:81-8.

[11] Gordon DB, Dahl JL, Miaskowski C McCarberg B, Todd KH, Paice JA, Lipman AG, Bookbinder M, Sanders SH Turk DC, Carr DB. American pain society recommendations for improving the quality of acute and cancer pain management: American Pain Society Quality of Care Task Force. Arch Intern Med 2005;165:1574-80.

[12] Green CR, Anderson KO, Baker TA, Campbell LC, Decker S, Fillingim RB, Kalauokalani DA, Lasch KE, Myers C, Tait RC, Todd KH, Vallerand AH. The unequal burden of pain: confronting racial and ethnic disparities in pain. Pain Med 2003;4:277-94.

[13] Greenland S, Senn SJ, Rothman KJ, Carlin JB, Poole C, Goodman SN, Altman DG. Statistical tests, $\mathrm{P}$ values, confidence intervals, and power: a guide to misinterpretations. Eur J Epidemiol 2016;31:337-50.

[14] Hadjistavropoulos T, Herr K, Turk DC, Fine PG, Dworkin RH, Helme R, Jackson K, 
Parmelee PA, Rudy TE, Lynn Beattie B, Chibnall JT, Craig KD, Ferrell B, Ferrell B, Fillingim RB, Gagliese L, Gallagher R, Gibson SJ, Harrison EL, Katz B, Keefe FJ, Lieber SJ, Lussier D, Schmader KE, Tait RC, Weiner DK, Williams J. An interdisciplinary expert consensus statement on assessment of pain in older persons. Clin J Pain 2007;23:S1-43.

[15] Hunnicutt JN, Tjia J, Lapane KL. Hospice use and pain management in elderly nursing home residents with cancer. J Pain Symptom Manage 2016. Published online ahead of print.

[16] IOM, Relieving pain in America: a blueprint for transforming prevention, care, education, and research. The National Academies Press;2011.

[17] Kaye AD, Baluch A, Scott JT. Pain management in the elderly population: a review. Ochsner J 2010;10:179-87.

[18] Lapane KL, Quilliam BJ, Chow W, Kim MS. Impact of revisions to the F-Tag 309 surveyors' interpretive guidelines on pain management among nursing home residents. Drugs Aging 2012;385-93.

[19] Lapane KL, Quilliam BJ, Chow W, Kim MS. The association between pain and measures of well-being among nursing home residents. J Am Med Dir Assoc 2012;13:344-9.

[20] Lapane KL, Quillaim BJ, Chow W, Kim MS. Pharmacologic management of non-cancer pain among nursing home residents. J Pain Symptom Manage 2013;45:33-42.

[21] Lin WC, Lum TY, Mehr DR, Kane RL. Measuring pain presence and intensity in nursing home residents. J Am Med Dir Assoc 2006;7:147-53.

[22] Lukas A Mayer B, Fialova D, Topinkova E, Gindin J, Onder G, Bernabei R, Nikolaus T, Denkinger MD. Pain Characteristics and Pain Control in European Nursing Homes: 
Cross-sectional and Longitudinal Results From the Services and Health for Elderly in Long TERm care (SHELTER) Study. J Am Med Dir Assoc 2013;14:421-8.

[23] Miller SC, Mor V, Wu N, Gozalo P, Lapane K. Does receipt of hospice care in nursing homes improve the management of pain at the end of life? J Am Geriatr Soc 2002;50:507-15.

[24] Miller SC, Lima J, Gozalo PL, Mor V. The growth of hospice care in U.S. nursing homes. J Am Geriatr Soc 2010;58:1481-8.

[25] Morris JN, Fries BE, Mehr DR, Hawes C, Phillips C, Mor V, Lipsitz LA. MDS cognitive performance scale. J Gerontol 1994;49:M174-82.

[26] Morris JN, Fries BE, Morris SA. Scaling ADLs within the MDS. J Gerontol A Biol Sci Med Sci 1999;54:M546-53

[27] Mossey JM. Defining racial and ethnic disparities in pain management. Clin Orthop Relat Res 2011;469:1859-70.

[28] Myers CD, Rileey JL, Robinson ME. Psychosocial contributions to sex-correlated differences in pain. Clin J Pain 2003;19:225-32.

[29] Pautex S, Herrmann F, Le Lous P, Fabjan M, Michel JP, Golg G. Feasibility and reliability of four pain self-assessment scales and correlation with an observational rating scale in hospitalized elderly demented patients. J Gerontol A Biol Sci Med Sci 2005;60:524-9.

[30] Pautex S, Michon A, Guedira M, Emond H, Le Lous P, Samaras D, Michel JP, Hermann F, Giannakopoulos P, Gold G. Pain in severe dementia: self-assessment or observational scales? J Am Geriatr Soc 2006;54:1040-5. 
[31] Pimentel CB, Briesacher BA, Gurwitz JH, Rosen AB, Pimentel MT, Lapane KL. Pain management in nursing home residents with cancer. J Am Geriatr Soc 2015;63:633-641.

[32] Pimentel CB, Gurwitz JH, Tjia J, Hume AL, Lapane KL. New initiation of long-acting opioids in long-stay nursing home residents. J Am Geriatr Soc 2016;64:1772-8.

[33] Portenoy RK. Treatment of cancer pain. Lancet 2011;377:2236-47.

[34] J.A. Sabin, A.G. Greenwald, The influence of implicit bias on treatment recommendations for 4 common pediatric conditions: pain, urinary tract infection, attention deficit hyperactivity disorder, and asthma., Am. J. Public Health. 102 (2012) 988-95.

[35] Saliba D, Buchanan J. Development and validation of a revised nursing home assessment tool: MDS 3.0. Rand Corp. Santa Monica, 2008.

[36] Saliba D, Buchanan J. Making the investment count: revision of the Minimum Data Set for nursing homes, MDS 3.0. J Am Med Dir Assoc 2012;13:602-610.

[37] Saliba D, Buchanan J, Edelen MO, Streim J, Ouslander J, Berlowitz D, Chodosh J. MDS 3.0: brief interview for mental status. J Am Med Dir Assoc 2012;13:611-7.

[38] Saliba D, Jones M, Streim J, Ouslander J, Berlowitz D, Buchanan J. Overview of significant changes in the Minimum Data Set for nursing homes version 3.0. J Am Med Dir Assoc 2012;13:595-601.

[39] Shavers VL, Bakos A, Sheppard VB. Race, ethnicity, and pain among the US adult population. J Health Care Poor Underserved 2010;21:177-220.

[40] Shen X, Zucerkman IH, Palmer JB, Stuart B. Trends in prevalence for moderate-tosevere pain and persistent pain among medicare beneficiaries in nursing homes, 20062009. J Gerontol A Biol Sci Med Sci 2015;70:598-603. 
[41] Simoni-Wastila L, Stuart BC, Shaffer T. Over-the-counter drug use by Medicare beneficiaries in nursing homes: implications for practice and policy. J Am Geriatr Soc 2006;54:1543-9.

[42] Takai Y, Yamamoto-Mitani N, Okamoto Y, Koyama K, Honda A. Literature review of pain prevalence among older residents of nursing homes. Pain Manag Nurs 2010;11:209-23.

[43] Teno JM, Kabumoto G, Wetle T, Roy J, Mor V. Daily pain that was excruciating at some time in the previous week: prevalence, characteristics, and outcomes in nursing home residents. J Am Geriatr Soc 2004;52:762-7.

[44] Teno JM, Gozalo PL, Bynum JP, Leland NE, Miller SC, Morden NE, Scupp T, Goodman DC, Mor V. Change in end-of-life care for Medicare beneficiaries: site of death, place of care, and health care transitions in 2000, 2005, and 2009. JAMA 2013;309:470-7.

[45] Teno JM, Weitzen S, Wetle T, Mor V. Persistent pain in nursing home residents. JAMA $2001 ; 285: 2081$.

[46] Thomas KS, Wysocki A, Intrator O, Mor V. Finding Gertrude: the resident's voice in Minimum Data Set 3.0. J Am Med Dir Assoc 2014;15:802-6.

[47] Tolson D, Rolland Y, Andriu S, Aquino JP, Beard J, Benetos A, Berrut G, Coll-Planas L, Dong B, Forette F, Franco A, Franzoni S, Salva A, Swagerty D, Trabucci M, Vellas B, Volicer L, Morley JE. International Association of Gerontology and Geriatrics: A Global Agenda for Clinical Research and Quality of Care in Nursing Homes, J Am Med Dir Assoc 2011;12:184-9.

[48] Turk DC, Wilson HD, Cahana A. Treatment of chronic non-cancer pain. Lancet 2011;377:2226-35. 
[49] Wei YJ, Simoni-Wastila L, Zuckerman IH, brandt N, Lucas JA. Algorithm for identifying nursing home days using Medicare claims and Minimum Data Set assessment data. Med Care 2016;54;e73-e77.

[50] Williams RL. A note on robust variance estimation for cluster-correlated data. Biometrics 2000;56:645-6.

[51] Won A, Lapane K, Gambassi G, Bernabei R, Mor V, Lipsitz LA. Correlates and management of nonmalignant pain in the nursing home. SAGE Study Group. Systematic Assessment of Geriatric drug use via Epidemiology. J Am Geriatr Soc 1999;47:936-42.

[52] Won AB, Lapane KL, Vallow S, Schein J, Morris JN, Lipsitz LA. Persistent nonmalignant pain and analgesic prescribing patterns in elderly nursing home residents. J Am Geriatr Soc $2004 ; 52: 867-74$

[53] Won A, Lapane KL, Vallow S, Schein J, Morris JN, Lipsitz LA. Long-term effects of analgesics in a population of elderly nursing home residents with persistent nonmalignant pain. J Gerontol A Biol Sci Med Sci 2006;61:165-9.

[54] Yong HH. Can attitudes of stoicism and cautiousness explain observed age-related 
variation in levels of self-rated pain, mood disturbance and functional interference in

chronic pain patients? Eur J Pain 2006;10:399-407.

[55] Zou G. A modified poisson regression approach to prospective studies with binary data. Am J Epidemiol 2004;159:702-6.

[56] Zou GY, Donner A. Extension of the modified Poisson regression model to prospective studies with correlated binary data. Stat Methods Med Res 2013;22:661-70. 


\section{FIGURE LEGENDS}

Figure 1. Variation in the crude prevalence of persistent and intermittent pain by age, gender, race/ethnicity, cognitive impairment, and cancer.

Figure 2. Pharmacologic pain management in long-stay nursing home residents by pain recorded at index assessment and in residents with persistent pain. 
Table 1. Resident characteristics and correlates by pain reported in long-stay United States nursing home residents $(\mathrm{N}=\mathbf{1}, \mathbf{3 8 7}, \mathbf{4 0 5})$.

\begin{tabular}{|c|c|c|c|c|c|}
\hline \multirow[b]{2}{*}{ Characteristics $^{a}$} & \multicolumn{3}{|c|}{ Pain Frequency } & \multirow{2}{*}{$\begin{array}{c}\text { Persistent pain } \\
\text { vs. no pain } \\
\begin{array}{c}\text { Adjusted } \\
(95 \% \mathrm{Cl})\end{array} \\
\end{array}$} & \multirow{2}{*}{$\begin{array}{c}\text { Intermittent } \\
\text { pain } \\
\text { vs. no pain } \\
\begin{array}{c}\text { Adjusted } \\
\text { (95 OR }\end{array} \\
(95 \% \mathrm{Cl})\end{array}$} \\
\hline & $\begin{array}{c}\text { Persistent } \\
\text { Pain } \\
(\mathrm{n}=270,244) \\
\end{array}$ & $\begin{array}{c}\text { Intermittent } \\
\text { Pain } \\
(\mathrm{n}=265,982) \\
\end{array}$ & $\begin{array}{c}\text { No } \\
\text { Pain } \\
(\mathrm{n}=851,179)\end{array}$ & & \\
\hline \multicolumn{6}{|l|}{ Age, years } \\
\hline $18-64$ & 19.4 & 12.5 & 12.7 & 1.07 (1.04-1.09) & $0.89(0.87-0.91)$ \\
\hline $65-74$ & 16.6 & 13.3 & 12.6 & Reference & Reference \\
\hline $75-84$ & 27.1 & 27.7 & 27.0 & $0.88(0.86-0.89)$ & $1.03(1.02-1.05)$ \\
\hline$\geq 85$ & 36.9 & 46.4 & 47.7 & $0.68(0.66-0.69)$ & $0.96(0.94-0.98)$ \\
\hline Women $^{c}$ & 71.7 & 70.1 & 66.3 & $1.55(1.53-1.57)$ & $1.23(1.21-1.24)$ \\
\hline \multicolumn{6}{|l|}{ Race/Ethnicity } \\
\hline Non-Hispanic White & 84.2 & 82.4 & 76.9 & Reference & Reference \\
\hline Non-Hispanic Black & 10.8 & 11.7 & 15.1 & $0.61(0.60-0.63)$ & $0.75(0.73-0.76)$ \\
\hline Hispanic & 3.5 & & 5.4 & $0.66(0.63-0.69)$ & $0.80(0.78-0.83)$ \\
\hline Other & 1.5 & & 2.6 & $0.54(0.50-0.58)$ & $0.67(0.64-0.71)$ \\
\hline \multicolumn{6}{|l|}{ Physical functioning $^{d}$} \\
\hline Moderate impairment & 47.4 & 49.2 & 47.5 & $1.06(1.04-1.08)$ & $1.12(1.10-1.13)$ \\
\hline Severe impairment & 26.5 & 29.4 & 31.7 & $1.10(1.08-1.13)$ & 1.05 (1.03-1.07) \\
\hline \multicolumn{6}{|l|}{ Cognitive functioning ${ }^{d}$} \\
\hline Moderate impairment & 27.6 & 30.4 & 29.5 & $0.48(0.47-0.49)$ & $0.67(0.66-0.68)$ \\
\hline Severe impairment & 22.9 & 47.4 & 50.3 & $0.26(0.25-0.26)$ & $0.48(0.48-0.49)$ \\
\hline Reject care $^{\mathrm{e}}$ & 11.3 & 11.6 & 11.9 & 1.19 (1.17-1.22) & 1.08 (1.06-1.09) \\
\hline Hospice use $e^{e}$ & 8.4 & 9.7 & 6.7 & $1.81(1.77-1.85)$ & $1.62(1.59-1.65)$ \\
\hline \multicolumn{6}{|l|}{ Comorbidities $^{\mathrm{e}}$} \\
\hline Dementia & 38.4 & 53.0 & 64.7 & $0.55(0.55-0.56)$ & $0.71(0.70-0.72)$ \\
\hline Cancer & 8.8 & 8.5 & 6.8 & $1.20(1.18-1.22)$ & $1.16(1.14-1.18)$ \\
\hline Arthritis & 35.8 & 31.4 & 23.6 & $1.91(1.88-1.94)$ & $1.46(1.44-1.48)$ \\
\hline Osteoporosis & 18.1 & 18.1 & 16.3 & $1.01(1.00-1.03)$ & $1.01(1.00-1.02)$ \\
\hline Fracture & 5.4 & 4.9 & 2.4 & 2.25 (2.19-2.32) & 1.98 (1.93-2.03) \\
\hline Pressure ulcers & 10.0 & 8.8 & 5.3 & $2.12(2.08-2.17)$ & $1.73(1.69-1.76)$ \\
\hline Surgical wounds & 3.1 & 2.1 & 0.9 & 2.68 (2.58-2.79) & $2.13(2.05-2.22)$ \\
\hline Staff-assessed pain at & 12.7 & 21.2 & 24.8 & $0.76(0.74-0.78)$ & $1.03(1.03-1.05)$ \\
\hline
\end{tabular}


index assessment

Cl: confidence interval; OR: odds ratio

${ }^{a}$ Characteristics are percentages unless otherwise noted. Columns may not add up to $100 \%$ due to rounding

${ }^{\mathrm{b}}$ Adjusted for all resident characteristics listed in table using a multinomial logistic model

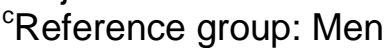

${ }^{\mathrm{d}}$ Reference group: No/mild impairment

${ }^{\mathrm{e}}$ Reference group: not having the behavior, service, or comorbidity (e.g., hospice use vs. no use). 
Table 2. Correlates of untreated and potentially undertreated pain in long-stay nursing home residents with persistent pain $(n=270,244)$.

\begin{tabular}{|c|c|c|c|c|}
\hline \multirow[b]{2}{*}{ Characteristic } & \multicolumn{2}{|c|}{ Untreated Pain $^{\mathrm{a}}$} & \multicolumn{2}{|c|}{ Potentially Undertreated Pain ${ }^{\mathrm{a}}$} \\
\hline & $\%$ & $\begin{array}{c}\text { Adjusted }^{b} \text { PR } \\
(95 \% \mathrm{Cl})\end{array}$ & $\%$ & $\begin{array}{c}\text { Adjusted }^{b} \text { PR } \\
(95 \% \mathrm{Cl})\end{array}$ \\
\hline \multicolumn{5}{|l|}{ Age, years } \\
\hline $18-64$ & 4.7 & $0.84(0.79-0.89)$ & 33.8 & $0.95(0.93-0.97)$ \\
\hline $65-74$ & 5.4 & Reference & 33.4 & Reference \\
\hline $75-84$ & 6.5 & $1.20(1.14-1.26)$ & 32.4 & $1.01(0.98-1.03)$ \\
\hline$\geq 85$ & 7.6 & $1.42(1.35-1.49)$ & 30.1 & $0.97(0.95-0.99)$ \\
\hline Women $^{c}$ & 6.1 & $0.76(0.74-0.79)$ & 30.6 & $0.91(0.90-0.93)$ \\
\hline \multicolumn{5}{|l|}{ Race/Ethnicity } \\
\hline Non-Hispanic White & 6.3 & Reference & 30.7 & Reference \\
\hline Non-Hispanic Black & 7.1 & $1.19(1.13-1.25)$ & 39.2 & $1.23(1.20-1.25)$ \\
\hline Hispanic & 5.7 & $0.87(0.80-0.95)$ & 38.9 & $1.18(1.14-1.22)$ \\
\hline Other & 7.5 & $1.15(1.03-1.29)$ & 36.1 & $1.11(1.05-1.17)$ \\
\hline \multicolumn{5}{|l|}{ Physical functioning } \\
\hline No / minor impairment & 6.6 & Reference & 33.2 & Reference \\
\hline Moderate impairment & 6.5 & $0.93(0.90-0.96)$ & 31.8 & $0.96(0.95-0.98)$ \\
\hline Severe impairment & 6.0 & $0.85(0.81-0.89)$ & 31.3 & $0.93(0.91-0.94)$ \\
\hline \multicolumn{5}{|l|}{ Cognitive functioning } \\
\hline No / minor impairment & 5.1 & Reference & 30.6 & Reference \\
\hline Moderate impairment & 6.9 & $1.24(1.19-1.29)$ & 33.1 & $1.11(1.09-1.12)$ \\
\hline Severe impairment & 8.5 & $1.51(1.44-1.57)$ & 33.6 & $1.14(1.11-1.16)$ \\
\hline Reject care $^{d}$ & 6.8 & $1.03(0.98-1.08)$ & 33.3 & $1.02(1.00-1.04)$ \\
\hline Hospice use $^{d}$ & 3.7 & $0.50(0.47-0.54)$ & 23.8 & $0.71(0.69-0.73)$ \\
\hline \multicolumn{5}{|l|}{ Comorbidities $^{d}$} \\
\hline Dementia & 7.8 & $1.16(1.12-1.20)$ & 31.8 & $1.00(0.99-1.02)$ \\
\hline Cancer & 5.0 & $0.81(0.76-0.86)$ & 29.3 & $0.93(0.91-0.96)$ \\
\hline Arthritis & 5.2 & $0.69(0.66-0.71)$ & 25.2 & $0.73(0.72-0.74)$ \\
\hline Osteoporosis & 5.7 & $0.92(0.88-0.96)$ & 26.2 & $0.87(0.85-0.89)$ \\
\hline Fracture & 4.6 & $0.72(0.67-0.78)$ & 34.9 & $1.14(1.10-1.17)$ \\
\hline Pressure ulcers & 4.4 & $0.70(0.66-0.75)$ & 29.5 & $0.90(0.88-0.92)$ \\
\hline Surgical wounds & 3.2 & $0.58(0.51-0.65)$ & 36.6 & $1.11(1.07-1.15)$ \\
\hline $\begin{array}{l}\text { Staff-assessed pain at } \\
\text { index assessment }^{d}\end{array}$ & 7.6 & $1.05(1.00-1.10)$ & 34.4 & $1.09(1.07-1.11)$ \\
\hline \multicolumn{5}{|c|}{$\begin{array}{l}\text { Cl: confidence interval; PR: prevalence ratio } \\
\text { aUntreated pain defined as receiving no analgesics at the index assessment. Potentially undertreated } \\
\text { pain defined as receiving no scheduled analgesics at the index assessment. } \\
\text { bAdjusted for all resident characteristics listed in table using modified Poisson models [56]. } \\
{ }^{\circ} \text { Reference group: Men }\end{array}$} \\
\hline
\end{tabular}




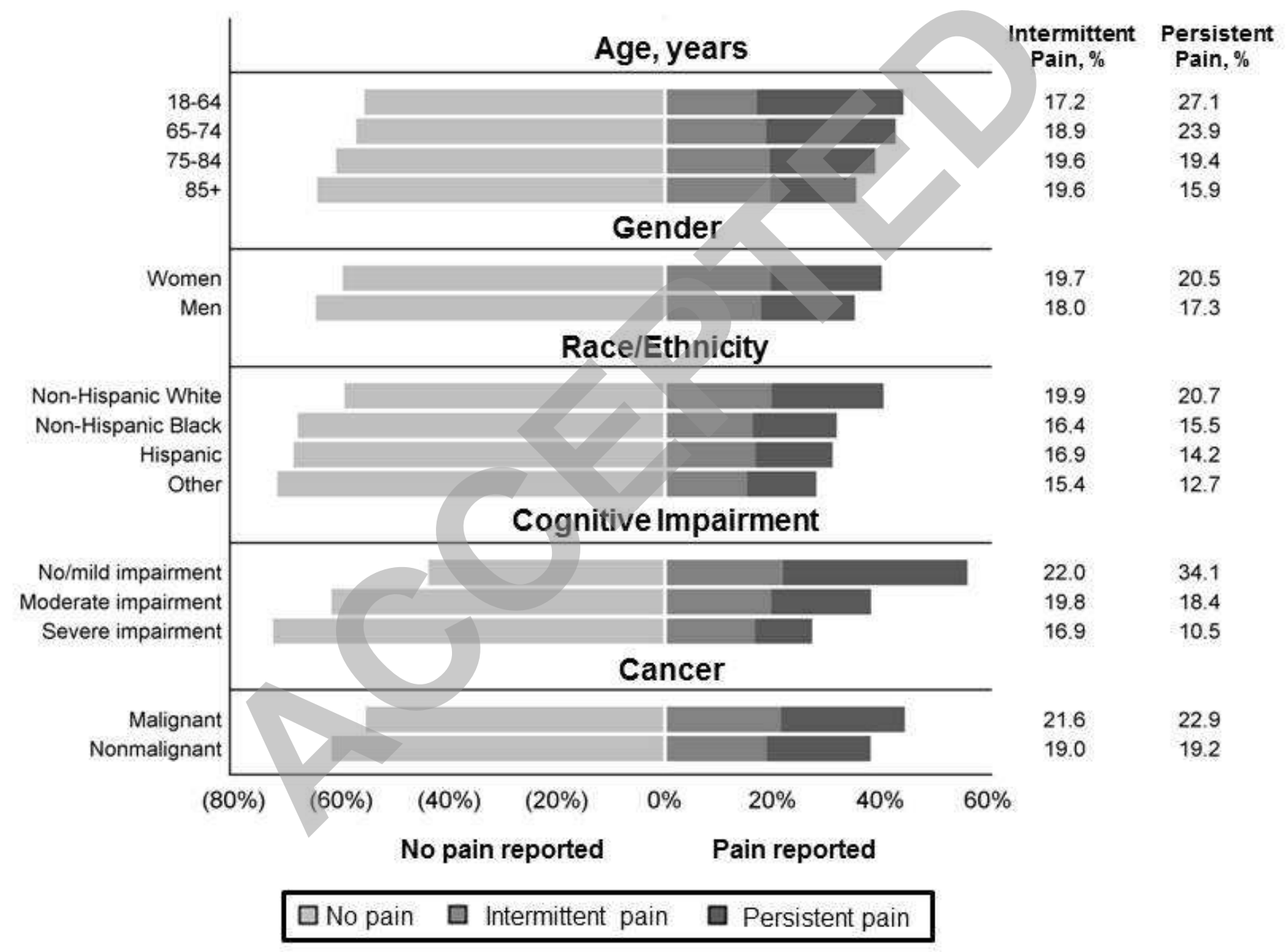




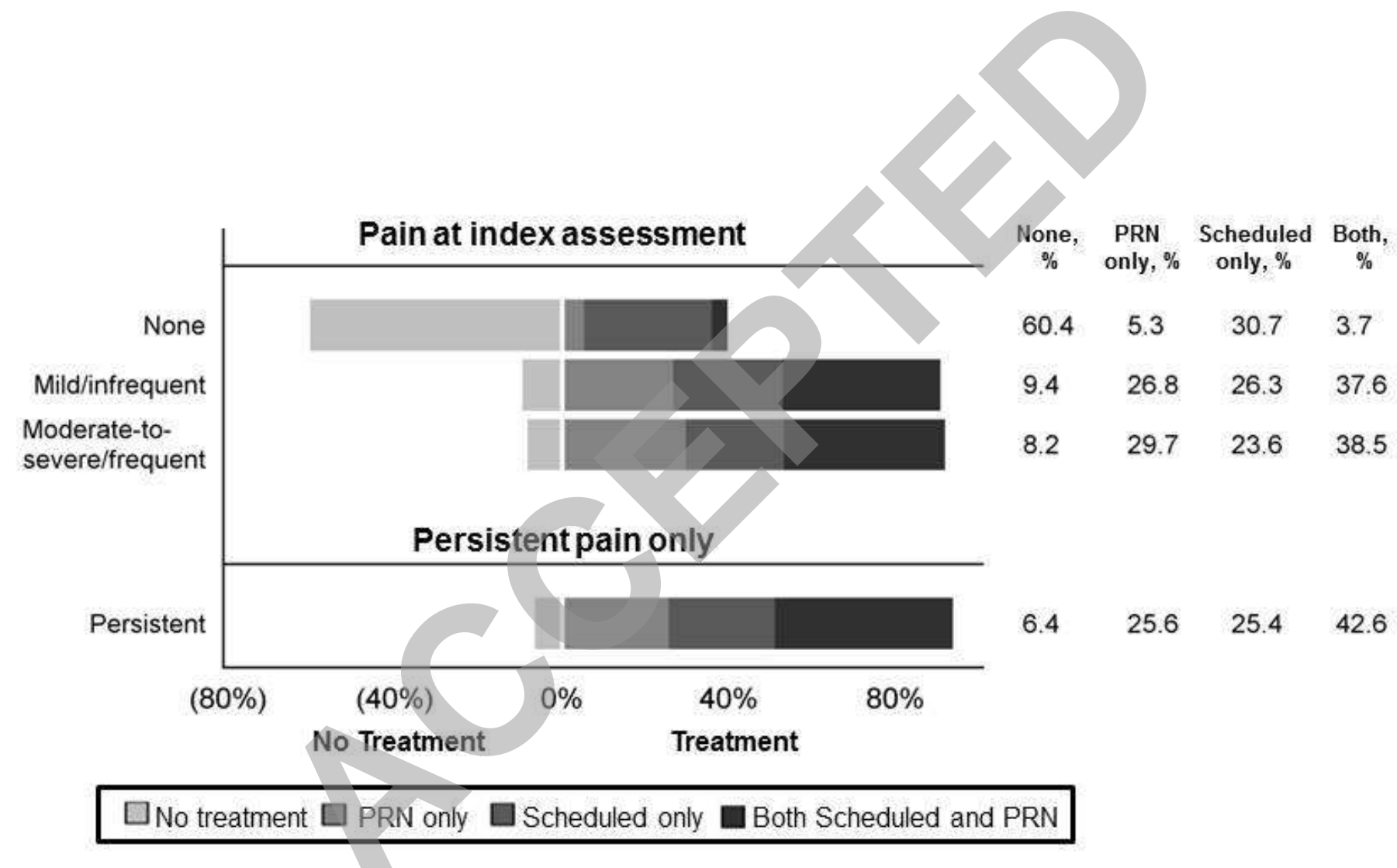

